\title{
Worse treatment response to neoadjuvant chemoradiotherapy in young patients with locally advanced rectal cancer
}

\author{
Yiyi Zhang ${ }^{1 \dagger}$, Liangliang $\mathrm{Yan}^{2 \dagger}$, Yong $\mathrm{Wu}^{1 \dagger}$, Meifang $\mathrm{Xu}^{3}$, Xing Liu ${ }^{1 *}$ and Guoxian Guan ${ }^{1 *}$
}

\begin{abstract}
Background: To evaluate the impact of age on the efficacy of neoadjuvant chemoradiotherapy (NCRT) in patients with locally advanced rectal cancer (LARC).

Method: LARC patients undergoing NCRT and radical surgery from 2011 to 2018 were divided into young $(<40$ years) and old ( $\geq 40$ years) groups. Multivariate analyses were performed to identify predictive factors for pathological complete response ( $p C R$ ). Predictive nomograms and decision curve analysis were used to compare the models including/excluding age groups. Immunohistochemical analysis was performed to detect CD133 expression in LARC patients.

Result: A total of 901 LARC patients were analyzed. The young group was associated with poorly differentiated tumors, more metastatic lymph nodes, higher perineural invasion, and a lower tumor regression grade $(P=0.008$; $P<0.001 ; P<0.001 ; P=0.003)$. Logistic regression analysis demonstrated that age $<40$ years $(H R=2.190, P=0.044)$, tumor size ( $H R=0.538, P<0.001)$, pre-NCRT $C N$ stage $(H R=0.570, P=0.036)$, and post-NCRT CEA level $(H R=0.877$, $P=0.001$ ) were significantly associated with $\mathrm{pCR}$. Predictive nomograms and decision curve analysis demonstrated that the predictive ability of models including the age group was superior to that of models excluding the age group. Higher CD133 expression was more common in young LARC patients.

Conclusion: Young patients with LARC were associated with lower pCR rates following NCRT. The ability of the predictive model was greater when based on the age group. Young LARC patients were associated with a higher CD133+ tumor stem cell burden, which contributed to the lower pCR rates.
\end{abstract}

Keywords: Age, pCR, Prognosis, LARC, CD133

\section{Background}

Colorectal cancer (CRC) is the third most common cancer and the second leading cause of cancer-related mortality in the USA [1]. CRC is generally thought to be a malignancy affecting the elderly patients. Over the last two decades, the incidence of CRC has increased in young individuals, especially those aged under 40.

\footnotetext{
*Correspondence: fjmufylx@163.com; fjxhggx@163.com

${ }^{\dagger}$ Yiyi Zhang, Liangliang Yan, and Yong Wu contributed equally to this work. ${ }^{1}$ Department of Colorectal Surgery, The First Affiliated Hospital of Fujian Medical University, Fuzhou, China

Full list of author information is available at the end of the article
}

However, most studies focus on older CRC patients, especially the elderly ( $>70$ years) $[2,3]$. Few studies have focused on the impact of young age ( $<40$ years) in CRC patients. In contrast to CRC in the elderly, young patients present at a more advanced tumor stage, with a more aggressive pathological subtype, and poor prognosis [4-6]. The increasing prevalence in CRC patients aged $<40$ years highlights a genuine need to better understand this disease in such patients.

Neoadjuvant chemoradiotherapy (NCRT) and radical resection have become the standard treatment for

C C The Author(s). 2020 Open Access This article is licensed under a Creative Commons Attribution 4.0 International License, which permits use, sharing, adaptation, distribution and reproduction in any medium or format, as long as you give appropriate credit to the original author(s) and the source, provide a link to the Creative Commons licence, and indicate if changes were made. The images or other third party material in this article are included in the article's Creative Commons licence, unless indicated otherwise in a credit line to the material. If material is not included in the article's Creative Commons licence and your intended use is not permitted by statutory regulation or exceeds the permitted use, you will need to obtain permission directly from the copyright holder. To view a copy of this licence, visit http://creativecommons.org/licenses/by/4.0/. The Creative Commons Public Domain Dedication waiver (http://creativecommons.org/publicdomain/zero/1.0/) applies to the data made available in this article, unless otherwise stated in a credit line to the data. 
patients with locally advanced rectal cancer (LARC). The well-documented benefits of this multimodality include a higher probability of tumor downsizing and downstaging, improved tumor resectability and sphincter preservation, and better local tumor control [7-9]. Approximately $10-30 \%$ of patients will achieve a pathological complete response (pCR), heralding an excellent prognosis due to low rates of local and distant recurrence $[7,10,11]$. Major efforts have been devoted to evaluating the influence of age on the efficacy and compliance of NCRT. While most studies were focused on old patients, few studies have focused on young LARC patients. Considering the aggressive tumor biology in young patients, we hypothesize that the efficacy of NCRT is reduced in young patients. Several recent studies have evaluated the impact of age on the efficacy of adjuvant chemotherapy in patients with rectal cancer [2, 12, 13]. Cancer stem cells (CSCs) have the capacity for multipotency and self-renewal and may be responsible for neoplasm formation, metastasis, recurrence, and therapeutic resistance [14-16]. Thus, we hypothesize that the early onset is due to higher malignancy cells, such as CSCs in young patients. Moreover, CSCs also indicate a poorer response to NCRT in LARC patients. However, data regarding the association of young age with treatment response to NCRT are scarce, and the influence of young age on the efficacy of NCRT in LARC patients remains unclear.

In this context, the present study was conducted to compare the efficacy of NCRT in young ( $<40$ years) and old ( $\geq 40$ years) patients with LARC in terms of tumor response. Additionally, we further investigated the relationship between young age and CSC (CD133 expression) frequency in LARC patients following NCRT.

\section{Patients and method Patient eligibility}

A retrospective study based on our prospectively maintained database was performed. LARC patients who underwent NCRT and radical resection between 2011 and 2018 were identified. The inclusion criteria were as follows: 1) clinical stage II or III (cT3/4 or cN1/2) disease; 2) pathologically proven rectal adenocarcinomas; and 3) tumors located within $12 \mathrm{~cm}$ from the anal verge. Exclusion criteria included: 1) previous of malignancies or concurrent with malignancies; 2) patients who underwent emergent surgery, palliative resection, or local excision. Finally, a total of 901 LARC patients were included. Since the majority of patients were pathologically confirmed by endoscopic biopsy when admitted to our hospital (a tertiary referral hospital), colonoscopy samples from only 169 patients were available for the immunohistochemical analysis. This study was approved by the Institutional Review Board (IRB) of Fujian
Medical University Union Hospital. A patient flow diagram was shown in Fig 1.

\section{Treatment protocol}

Comprehensive assessments for tumor staging of patients were performed by a digital rectal examination, colonoscopy, chest radiography, abdominopelvic magnetic resonance imaging (MRI), and/or transrectal ultrasound (ERUS). Preoperative long-course radiotherapy consisted of a total dose of $45 \mathrm{~Gy}$ to the pelvis, delivered in 25 fractions for 5 consecutive weeks ( 180 cGy per fraction, 5 days a week), followed by a boost of 5.4 Gy to the primary tumor. Preoperative concurrent chemotherapy was initiated on the first day of radiotherapy by using 5-FU plus oxaliplatin (FOLFOX) or capecitabine plus oxaliplatin (CapeOX).

Surgical operation was performed at an interval of 68 weeks after the completion of radiation. Surgical resection for rectal cancer was performed according to the principle of total mesorectal excision (TME) and high ligation of the inferior mesenteric artery. The surgical procedure consisted of low anterior resection (LAR), abdominoperineal resection (APR), or Hartmann's procedure. About 3-4 weeks after surgery, postoperative adjuvant chemotherapy was administered to patients (using FOLFOX or CapeOX) for 6 months.

\section{Definitions}

Tumor response to NCRT was graded according to the tumor regression grade (TRG) system [17]. pCR was defined as the absence of viable tumor cells in either the primary tumor site or the resected lymph nodes. Postoperative morbidity was classified according to the Clavien-Dindo classification, grades I-II was considered as minor complications, and grades III-V as major complications. Tumor size was divided according to the quartile intervals to make it more applicable in clinical practice $(\leq 1.8 \mathrm{~cm}, 1.9-3.1 \mathrm{~cm}, \geq 3.2 \mathrm{~cm})$. Perioperative mortality was defined as any death within 30 days of surgery or occurring in the hospital.

\section{Immunohistochemical analysis}

CD133 (Affinity Biosciences, AF5120, Polyclonal, 1:100) protein expression in specimens obtained before NCRT in 169 LARC patients was measured using the immunohistochemical streptavidin-biotin complex method (Fig. 3e, f, g, h) [18]. Phosphate-buffered saline (PBS) was used as the negative control and the image of the positive control from GE Healthcare Life Sciences. Immunoreactivity was scored by semi-quantitative analysis, and the fields were randomly selected in five directions (up, center, down, left, and right) under high magnification $(\times 400)$. The color was determined based on the intensity score as follows: 0 (no staining), 1 (light yellow), 


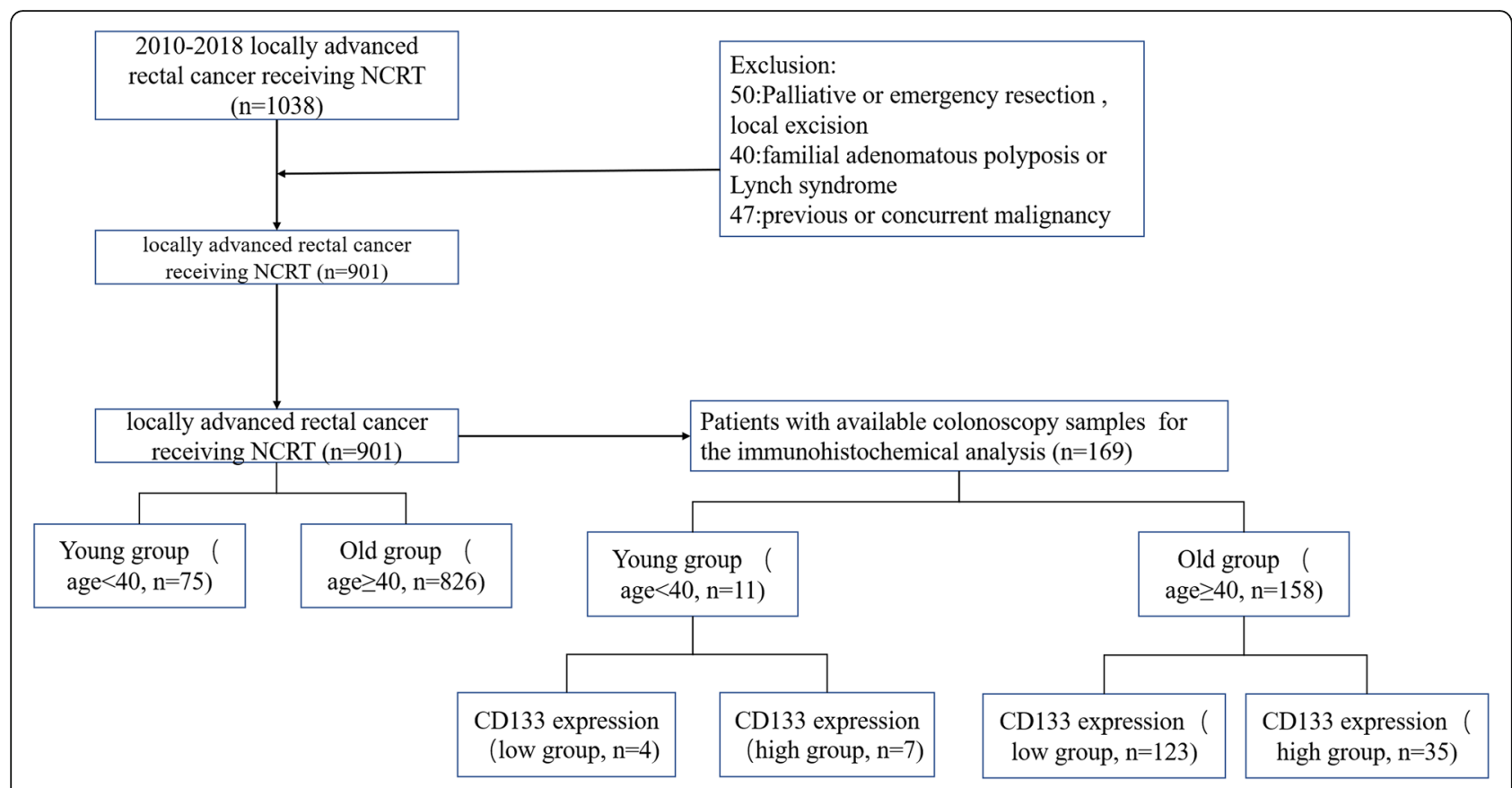

Fig. 1 Patient flow diagram

2 (brown), and 3 (deep brown). The percentage of positive cells was scored as $0(<5 \%), 1(5-25 \%), 2(25-50 \%)$, $3(50-75 \%)$, and $4(>75 \%)$. The mean value was calculated for each case with the aforementioned scoring methods and the final score was obtained by multiplying these two scores. All analyses were performed in a double-blinded manner.

\section{Statistical analysis}

Statistical analysis was performed using SPSS version 23.0 (SPSS INC., Chicago, USA) and R software packages, version 3.5.1 (The $\mathrm{R}$ Foundation for Statistical Computing, http://www.r-project.org/). The categorical variables were presented as frequencies and percentages and assessed using the Chi-squared $\left(\chi^{2}\right)$ test or Fisher's exact test. The continuous variables were described as means \pm standard deviation and assessed using Student's $t$-test. All significant variables in the univariate analysis were entered into a Logistic regression model to identify predictors of $\mathrm{pCR}$. Based on the multivariable analysis, a predictive nomogram was developed using the $\mathrm{R}$ project. The performance of the nomogram was evaluated by calculating the Harrell's concordance index (c-index). Decision curve analysis (DCA) was performed to evaluate the clinical utility of the model for pCR. DCA is a method for evaluation and comparison of the predictive value between different prediction models [19, 20]; therefore, this method was used to evaluate the clinical utility of the model for pCR. The $\mathrm{x}$-axis of the DCA represents the percentage of threshold probability, and the $y$-axis represents the net benefit of the predictive model. The net benefit was calculated according to the following formula: Net benefit $=($ true positives $/ \mathrm{n})-($ false positives $/ \mathrm{n}) *(\mathrm{pt} /(1-\mathrm{pt})$. "Number high risk" indicated the number of patients classified as positive (high risk) by a model including age group according to various threshold probabilities. "Number high risk with the event" was the true positive patient number according to various threshold probabilities. The optimal cut-off values for CD133 expression were calculated and determined by using the X-tile program (http://www.tissuearray.org/ rimmlab/), a new bio-informatics tool for biomarker assessment and outcome-based cut-point optimization, which identified the cut-off with the minimum $p$ values from log-rank $\chi^{2}$ statistics in terms of OS [21]. The optimal cut-off score was identified as 11 for CD133. Thus, we defined CD133 expression score $\leq 11$ as low expression, and $>11$ as high expression. Survival outcomes were assessed using the Kaplan-Meier method and logrank test. $P<0.05$ was considered to indicate statistical significance.

\section{Results}

\section{Patient characteristics}

A total of 901 LARC patients were eligible for our analysis. Among them, 75 (8.3\%) were assigned to the young group and $826(91.7 \%)$ patients were assigned to the old groups. The median ages in the two groups were 34.3 and 58.1 years, respectively. Additionally, the old group was associated with a higher American Society of 
Table 1 Patient characteristics in patients with LARC after NCRT

\begin{tabular}{|c|c|c|c|}
\hline Characteristics & Young group $(n=75)$ & Old group $(n=826)$ & $p$ value \\
\hline Sex (\%) & & & 0.527 \\
\hline Male & $52(69.3)$ & $537(65.0)$ & \\
\hline Female & $23(30.7)$ & $289(35.0)$ & \\
\hline Age (years) & $34.3 \pm 4.1$ & $58.1 \pm 9.4$ & $<0.001$ \\
\hline ASA score (\%) & & & $<0.001$ \\
\hline 1 & $75(100)$ & $603(73.0)$ & \\
\hline 2 & 0 & $209(25.3)$ & \\
\hline 3 & 0 & $14(1.6)$ & \\
\hline Distance from the anal verge $(\mathrm{cm})$ & $6.4 \pm 2.5$ & $6.3 \pm 2.3$ & 0.684 \\
\hline Time interval between CRT and surgery (weeks) & $9.1 \pm 1.4$ & $9.4 \pm 2.9$ & 0.468 \\
\hline Clinical T stage (\%) & & & 0.173 \\
\hline $\mathrm{T} 1$ & $1(1.3)$ & $2(0.2)$ & \\
\hline $\mathrm{T} 2$ & 0 & $18(2.2)$ & \\
\hline T3 & $25(33.3)$ & $317(38.4)$ & \\
\hline T4 & $49(65.3)$ & $489(59.7)$ & \\
\hline Clinical N stage (\%) & & & 1.000 \\
\hline No & $7(9.3)$ & $79(9.5)$ & \\
\hline $\mathrm{N}+$ & $68(90.7)$ & $747(90.5)$ & \\
\hline Pre-NCRT CEA level (\%) & & & 0.562 \\
\hline$<5.0 \mathrm{ng} / \mathrm{ml}$ & $25(33.3)$ & $283(34.3)$ & \\
\hline$\geq 5.0 \mathrm{ng} / \mathrm{ml}$ & $24(32.0)$ & $219(26.5)$ & \\
\hline Unknown & $26(34.7)$ & $324(39.2)$ & \\
\hline Pre-NCRT CA19-9 level (\%) & & & 0.641 \\
\hline$<39.0 \mathrm{U} / \mathrm{ml}$ & $40(53.3)$ & $430(52.1)$ & \\
\hline$\geq 39.0 \mathrm{U} / \mathrm{ml}$ & $9(12.0)$ & $76(9.2)$ & \\
\hline Unknown & $26(34.7)$ & $320(38.7)$ & \\
\hline Post-NCRT CEA level (\%) & & & 0.115 \\
\hline$<5.0 \mathrm{ng} / \mathrm{ml}$ & $67(89.3)$ & $672(81.4)$ & \\
\hline$\geq 5.0 \mathrm{ng} / \mathrm{ml}$ & $8(10.7)$ & $154(18.6)$ & \\
\hline Post-NCRT CA19-9 level (\%) & & & 0.320 \\
\hline$<39.0 \mathrm{U} / \mathrm{ml}$ & $68(90.7)$ & $775(93.8)$ & \\
\hline$\geq 39.0 \mathrm{U} / \mathrm{ml}$ & $7(9.3)$ & $51(6.2)$ & \\
\hline
\end{tabular}

LARC Locally advanced rectal cancer; NCRT Neoadjuvant chemoradiotherapy; ASA American Society of Anesthesiologists; CRT Chemoradiotherapy; CEA Carcinoembryonic Antigen; CA19-9 Carbohydrate Antigen 19-9

Anaesthesiology (ASA) grade $(P<0.05)$. As shown in Table 1, there were no significant differences between the two groups in terms of sex, the interval between NCRT and surgery, distance from the anal verge, clinical T stage, clinical N stage, pre-NCRT CA19-9 level, preNCRT CEA level, post-NCRT CA19-9 level, and postNCRT CEA level (all $P>0.05$ ),

\section{Perioperative, pathological and survival outcomes}

No significant differences were observed between the two groups in terms of estimated blood loss, operation time, surgical approach, peri-NCRT complication rates, and sphincter-saving procedure (all $P>0.05$, Table 2). There were no significant differences between the two groups in terms of postoperative hospital stay and postoperative complications $(P=0.124, P=0.736$, respectively). Similarly, the chemotherapy regimen did not differ between the two groups $(P=0.461)$. Compared to the young group, mucinous or signet ring cell carcinoma, $(17.3 \%$ vs. $7.6 \%, P=0.008)$ or poorly differentiated tumors $(24.3 \%$ vs. $9.0 \%, P<0.001)$ were more common in the old group. Moreover, the young group was associated with a higher TRG $(P=0.003)$, as well as a higher rate of perineural invasion $(17.3 \%$ vs. $6.5 \%, P=0.002)$. 
Table 2 Operative and postoperative outcomes in patients with LARC after NCRT

\begin{tabular}{|c|c|c|c|}
\hline Characteristics & Young group $(n=75)$ & Old group $(n=826)$ & $p$ value \\
\hline Operative time (min) & $232.5 \pm 81.2$ & $225.8 \pm 64.0$ & 0.394 \\
\hline Estimated blood loss (ml) & $77.7 \pm 74.2$ & $88.9 \pm 105.5$ & 0.372 \\
\hline Surgery approach (\%) & & & 0.882 \\
\hline Laparoscopic & $49(65.3)$ & $549(66.5)$ & \\
\hline Open & $18(24.0)$ & $198(23.9)$ & \\
\hline Robotic & $8(10.7)$ & $79(9.6)$ & \\
\hline Postoperative hospital stay (days) & $7.8 \pm 3.4$ & $8.8 \pm 5.5$ & 0.124 \\
\hline Postoperative complications (\%) & $12(16.0)$ & $122(14.9)$ & 0.736 \\
\hline 30 days readmission (\%) & $1(1.3)$ & $5(0.6)$ & 0.407 \\
\hline Peri-CRT complications ${ }^{\mathrm{a}}$ & $25(33.3)$ & 259 (31.4) & 0.699 \\
\hline Major & $1(1.3)$ & $23(2.8)$ & 0.741 \\
\hline Sphincter-saving procedure (\%) & $66(88.0)$ & $721(87.4)$ & 1.000 \\
\hline Pathological type (\%) & & & 0.936 \\
\hline Ulcering & $72(96.0)$ & $796(96.7)$ & \\
\hline Expanding & $1(1.3)$ & $11(1.2)$ & \\
\hline Infiltrating & $2(2.7)$ & $19(2.1)$ & \\
\hline Histopathology (\%) & & & 0.008 \\
\hline Adenocarcinoma & $62(82.7)$ & $763(92.4)$ & \\
\hline Mucinous or signet ring cell carcinoma & $13(17.3)$ & $63(7.6)$ & \\
\hline Tumor differentiation (\%) & & & $<0.001$ \\
\hline Well to moderately differentiated & $57(75.7)$ & $752(91.0)$ & \\
\hline Poorly differentiated and others & $18(24.3)$ & $74(9.0)$ & \\
\hline Chemotherapy regimen (\%) & & & 0.461 \\
\hline FOLFOX/CapeOX & $33(44.0)$ & $326(39.5)$ & \\
\hline Capecitabine & $42(56.0)$ & $500(60.5)$ & \\
\hline Lymph nodes retrieved & $19.3 \pm 13.1$ & $12.0 \pm 6.2$ & $<0.001$ \\
\hline Metastatic lymph nodes & $2.2 \pm 6.1$ & $0.7 \pm 2.0$ & $<0.001$ \\
\hline CRM involvement (\%) & $1(1.3)$ & $10(1.2)$ & 1.000 \\
\hline Tumor size $(\mathrm{cm})$ & & & 0.069 \\
\hline$\leq 1.8 \mathrm{~cm}$ & $17(22.7)$ & $212(25.7)$ & \\
\hline $1.9-3.1 \mathrm{~cm}$ & $31(41.3)$ & $416(50.4)$ & \\
\hline$\geq 3.2 \mathrm{~cm}$ & $27(36.0)$ & $198(24.0)$ & \\
\hline Pathological TNM stage (\%) & & & 0.957 \\
\hline 0 & $9(12.0)$ & $183(22.2)$ & \\
\hline । & $14(18.7)$ & $205(24.8)$ & \\
\hline$\|$ & $16(21.3)$ & $217(26.3)$ & \\
\hline III & $31(41.3)$ & $179(21.7)$ & \\
\hline IV & $5(6.7)$ & $42(5.2)$ & \\
\hline TRG grade (\%) & & & 0.003 \\
\hline 0 & $9(12.0)$ & $183(22.2)$ & \\
\hline 1 & $20(26.7)$ & $272(32.9)$ & \\
\hline 2 & $33(44.0)$ & $313(37.9)$ & \\
\hline 3 & $13(17.3)$ & $58(7.0)$ & \\
\hline Perineural invasion (\%) & $13(17.3)$ & $54(6.5)$ & 0.002 \\
\hline
\end{tabular}


Table 2 Operative and postoperative outcomes in patients with LARC after NCRT (Continued)

\begin{tabular}{llll}
\hline Characteristics & Young group $(n=75)$ & Old group $(n=826)$ & $p$ value \\
\hline Vascular invasion (\%) & $4(5.3)$ & $29(3.5)$ & 0.346 \\
Adjuvant chemotherapy (\%) & & & 0.484 \\
$\quad$ Complete & $44(58.7)$ & $427(51.7)$ \\
Decreased complete & $9(12.0)$ & $87(10.5)$ \\
Refuse & $2(2.7)$ & $44(5.3)$ \\
Unknown & $20(26.7)$ & $268(32.4)$ \\
\hline
\end{tabular}

a Some patients experienced more than one complication, and categorized as

NCRT Neoadjuvant chemoradiotherapy; CRM Circumferential resection margin; TRG Tumor regression grade

Pathological TNM stage and pathological type were similar in both groups $(P=0.957, P=0.936)$. Similarly, there were no differences between the two groups in terms of vascular invasion and tumor size did $(P=0.346$, $P=0.069$ ). A positive circumferential resection margin (CRM) was observed in one patient (1.3\%) in the young group, 10 patients $(1.2 \%)$ in the old group, with no significant difference between the two groups $(P=1.000)$. Moreover, Kaplan-Meier curve analysis demonstrated that young patients were associated with poorer prognosis in LARC patients following NCRT. The 3-year OS rate in the old group ( $\geq 40$ years) was significantly higher than that in the young group (<40 years) $(88.3 \%$ vs. $71.6 \% ; P=0.01$, Fig. $3 \mathrm{~m})$. Moreover, the 3 -year DFS rate for the old group ( $\geq 40$ years) was higher than that in the young group $(<40$ years $)(83.8 \%$ vs. $68.6 \% ; P=0.204$, Fig. 31).

\section{Association between young age and $\mathrm{pCR}$}

To explore the association between young age and treatment response to NCRT, we identified predictive factors for pCR by Logistic regression analysis. In the univariate analysis, tumor size $(\leq 1.8 \mathrm{~cm}$ vs. $1.9-3.1 \mathrm{~cm}$ OR $=6.764$, $P<0.001$; vs. $\geq 3.2 \mathrm{~cm} \mathrm{OR}=2.022, P=0.007)$, age $(\geq 40$ years vs. $<40$ years, $\mathrm{OR}=2.087, P=0.044$ ), pre-NCRT clinical $\mathrm{T}$ stage $(\mathrm{OR}=0.731, P=0.031)$, pre-NCRT clinical $\mathrm{N}$ stage $(\mathrm{OR}=0.550, P=0.016)$, distance from the anal verge (OR $=0.919, P=0.018)$, and post-NCRT CEA $(\mathrm{OR}=0.381, P<0.001)$ were significantly associated with pCR in LARC patients. In the multivariate analysis, tumor size $(\leq 1.8 \mathrm{~cm}$ vs. $1.9-3.1 \mathrm{~cm} \quad$ OR $=6.764, P<$

Table 3 Univariate and multivariate analysis of predictive factors for pCR in LARC patients $(n=901)$

\begin{tabular}{|c|c|c|c|c|c|c|}
\hline \multirow[t]{2}{*}{ Variables } & \multicolumn{3}{|c|}{ Univariate analysis } & \multicolumn{3}{|c|}{ Multivariate analysis } \\
\hline & $\overline{\mathrm{HR}}$ & $95 \% \mathrm{Cl}$ & $\overline{p \text { value }}$ & $\overline{\mathrm{HR}}$ & $95 \% \mathrm{Cl}$ & $p$ value \\
\hline Sex (male vs. female) & 1.351 & $0.973-1.875$ & 0.073 & & & \\
\hline Age ( $\geq 40$ years vs. $<40$ years) & 2.087 & $1.020-4.269$ & 0.044 & 2.382 & $1.105-5.137$ & 0.027 \\
\hline ASA & 0.964 & $0.686-1.353$ & 0.831 & & & \\
\hline Distance from the anal verge & 0.919 & $0.856-0.986$ & 0.018 & 0.949 & $0.878-1.025$ & 0.181 \\
\hline \multicolumn{7}{|l|}{ Tumor size } \\
\hline$\leq 1.8 \mathrm{~cm}$ & Reference & Reference & $<0.001$ & Reference & Reference & $<0.001$ \\
\hline $1.9-3.1 \mathrm{~cm}$ & 6.764 & $4.019-11.385$ & $<0.001$ & 5.806 & $3.412-9.878$ & $<0.001$ \\
\hline$\geq 3.2 \mathrm{~cm}$ & 2.022 & $1.212-3.373$ & 0.007 & 1.853 & $1.101-3.119$ & 0.020 \\
\hline Time interval between NCRT and surgery & 1.041 & $0.988-1.097$ & 0.131 & & & \\
\hline Pre-NCRT cT stage & 0.731 & $0.553-0.967$ & 0.031 & 0.816 & $0.607-1.097$ & 0.177 \\
\hline Pre-NCRT cN stage & 0.550 & $0.338-0.895$ & 0.016 & 0.560 & $0.332-0.943$ & 0.029 \\
\hline Post-NCRT CEA level & 0.381 & $0.224-0.647$ & $<0.001$ & 0.873 & $0.805-0.946$ & 0.001 \\
\hline Post-NCRT CA19-9 level & 0.492 & $0.219-1.101$ & 0.084 & & & \\
\hline Chemotherapy regimen & 1.083 & $0.783-1.499$ & 0.630 & & & \\
\hline Radication dose reduction & 0.978 & $0.360-2.653$ & 0.965 & & & \\
\hline NCRT complications & 0.900 & $0.641-1.264$ & 0.542 & & & \\
\hline
\end{tabular}

pCR Pathological complete response; NCRT Neoadjuvant chemoradiotherapy; HR Hazard ratio; Cl Confidential interval; ASA American Society of Anesthesiologists; CEA Carcinoembryonic Antigen; CA19-9 Carbohydrate Antigen 19-9 
0.001 ; vs. $\geq 3.2 \mathrm{~cm} \mathrm{OR}=2.022, P=0.007)$, age $(\geq 40$ years vs. $<40$ years, $\mathrm{OR}=2.382, P=0.027)$, pre-NCRT clinical $\mathrm{N}$ stage $(\mathrm{OR}=0.560, P=0.029)$, and post-NCRT CEA $(\mathrm{OR}=0.873, P=0.001)$ were independent predictive factors for pCR in LARC patients (Table 3).

Predictive nomogram for $\mathrm{PCR}$ and decision curve analysis By incorporating the significant determinants in the Logistic regression analysis, we developed a predictive nomogram for $\mathrm{pCR}$ in LARC patients after NCRT as shown in Fig. 2a. The c-index of the nomogram was 0.70 (95\% CI 0.70-0.71). The calibration curve (Fig. 2b) presented good statistical performance upon internal validation between predicted and actual $\mathrm{pCR}$ rates. DCA was used to evaluate the performance of the nomogram. As shown in Fig. 2c, the model including age group provided more predictive power than either the $\mathrm{pCR}$ scheme or the non-pCR scheme. The clinical impact curve (Fig. 2d) shows the prediction of risk stratification of 1000 patients using a resampling bootstrap method.

\section{The association of CD133 expression with survival, pCR} rate, and young age

We further investigated the reason for the lower $\mathrm{pCR}$ rates in young LARC patients by examining CSC frequency (based on CD133 expression, Fig. 3e, f, g and h). A total of 169 LARC patients were eligible for immunohistochemical analysis. Since the CD133 expression scores were continuous variables, the $\mathrm{X}$-tile program was utilized to identify the optimal cut-off points for determining the greatest actuarial survival difference. Using this method 11 was identified as the cut-off value for CD133 expression (Fig. 3a and b). Based on these cut-off points for CD133 expression, we divided the cohort into low $(n=127)$ and high $(n=42)$ subgroups in terms of OS and DFS.

Higher CD133 scores were associated with poorer prognosis in LARC patients following NCRT. The 3-year OS rate for the low CD133 group was significantly higher than that in the high CD133 group $(95.1 \%$ vs. 62.9\%; $P<0.01$, Fig. 3d). Moreover, lower CD133 scores were correlated with improved DFS (Fig. 3c). The 3-year DFS rate for the low CD133 group was significantly

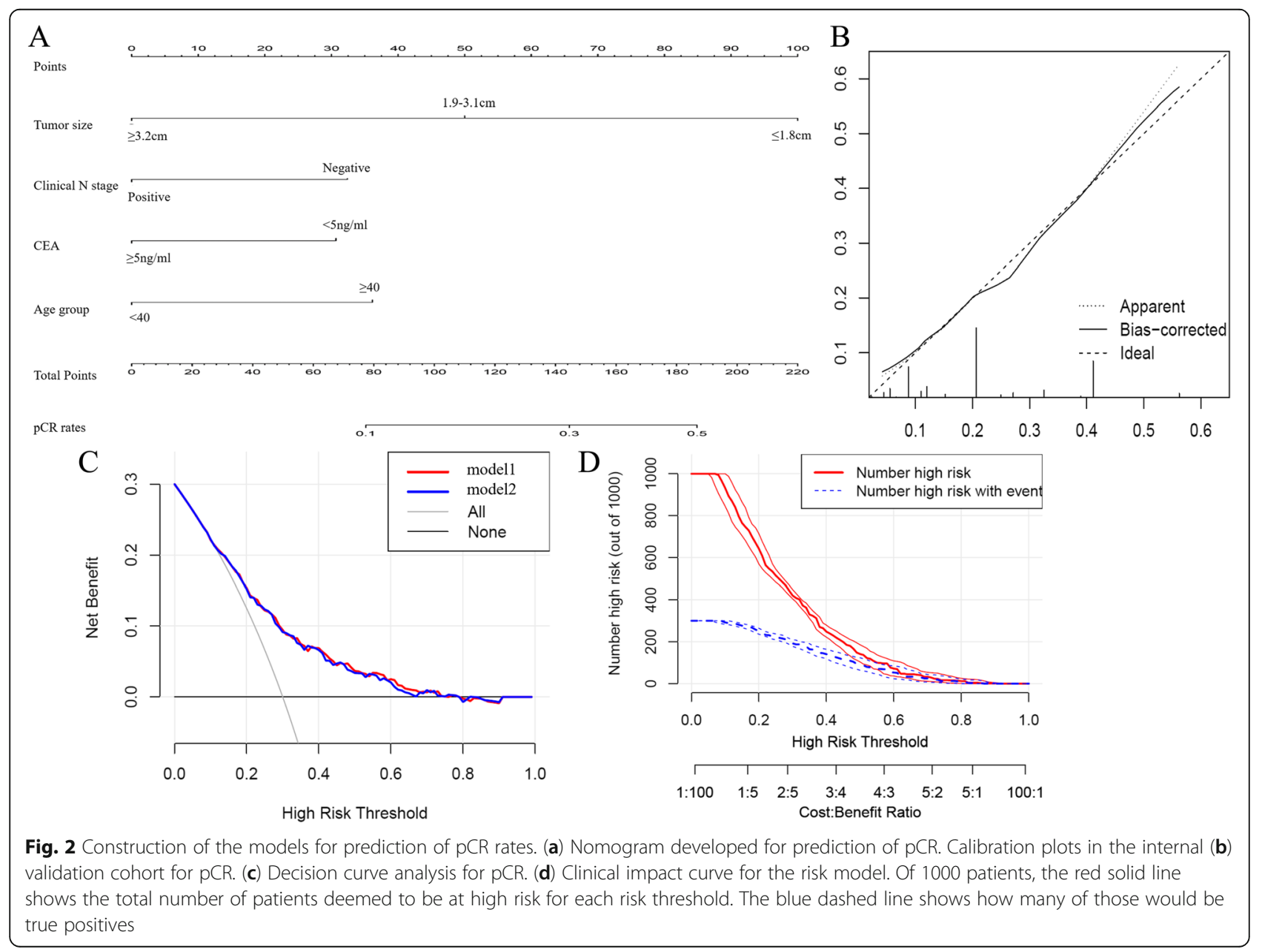




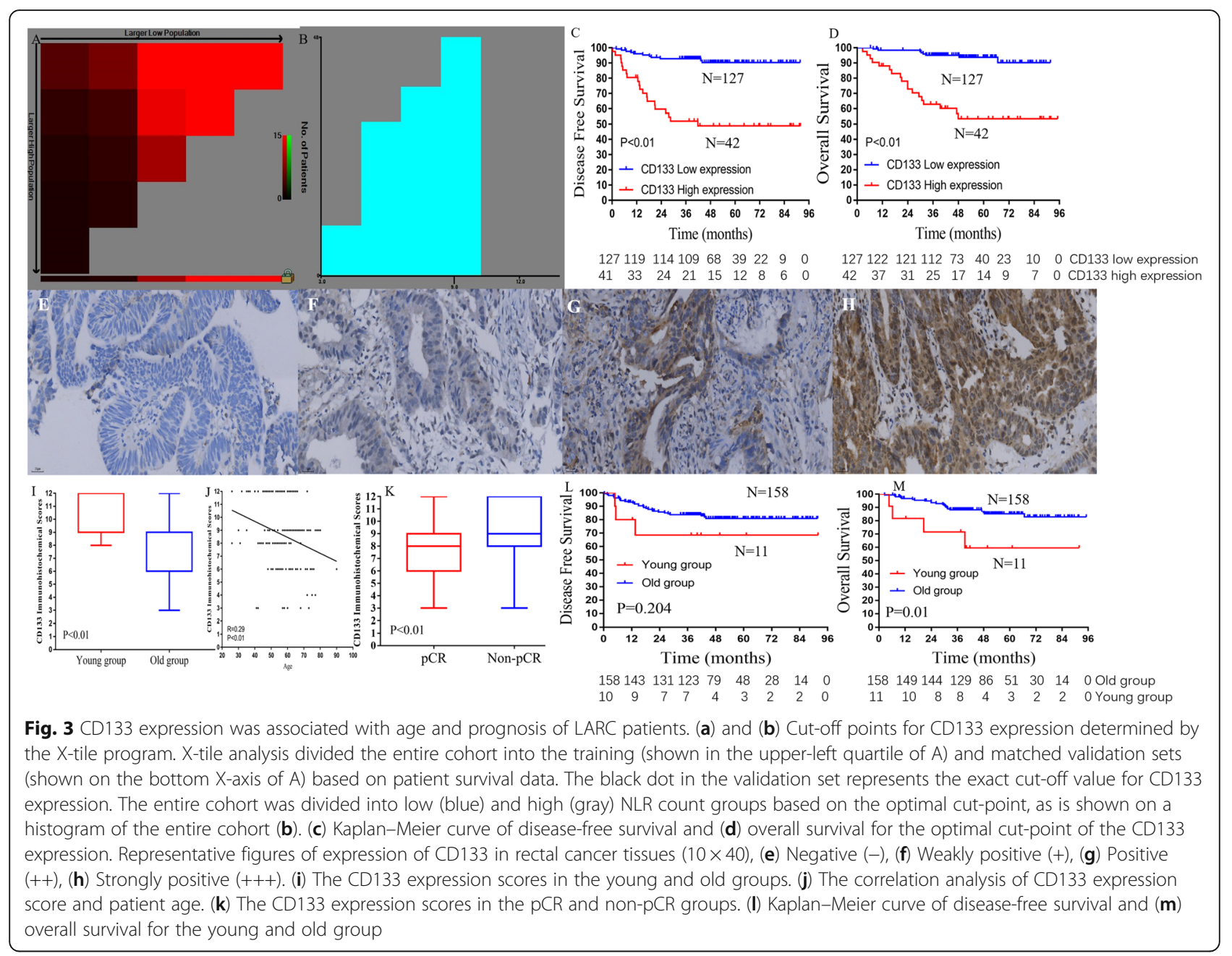

higher than that in the high CD133 group (92.8\% vs. $50.7 \% ; P<0.01$ ). As shown in Fig. 3i, young patients had a higher CD133 expression score compared to that of old patients $(P<0.01)$. Moreover, correlation analysis demonstrated that CD133 expression was associated with patient age $(P<0.01$, Fig. $3 j)$. Moreover, we examined the CD133 expression in the pCR and non-pCR groups, the results demonstrated that the CD133 expression value in the $\mathrm{pCR}$ group was significantly lower than in the non-pCR group $(P<0.01$, Fig. 3k). Taken together, these findings indicated that young LARC patients were associated with a higher CD133+ CSC burden, which might contribute to the lower $\mathrm{pCR}$ rates.

\section{Discussion}

Age is an important factor affecting the efficacy of NCRT in rectal cancer patients. Few studies have focused on young LARC patients following NCRT. In the present study, we explored the efficacy of NCRT in young ( $<40$ years) and old ( $\geq 40$ years) LARC patients. The results demonstrated a lower $\mathrm{pCR}$ rate in young
LARC patients compared with that in old patients, without affecting postoperative complications. For the first time, our study demonstrated that young patients have a higher proportion of CSCs (CD133+), which might contribute to the lower $\mathrm{pCR}$ rates.

Young LARC patients often present with aggressive pathological features and advanced stage compared with older patients [4-6]. Additionally, aggressive pathological features could result in a poorer response to NCRT [22-28]. Our results are consistent with those reported by Li et.al [29], in which analysis of the Surveillance, Epidemiology, and End Results (SEER) population-based database revealed that young patients had a better prognosis than old patients. However, the prognosis was inconsistent with the pathological results in their study, because young patients had more aggressive pathological features compared with old patients. This discrepancy may be explained that by the lack of cancer therapy information, including neoadjuvant and adjuvant treatment, and quality of surgery, in the SEER database, all of which are factors that play an important 
role in patient survival outcome. When treating young LARC patients with rectal cancer, it is important to determine how aggressive the tumor is, so that patients can be informed about the advantages and disadvantages of the treatment. In our study, we demonstrated that young LARC patients displayed poorer pathological features, such as a higher probability of mucinous or signet ring cell and poorly differentiated tumors, which is in accordance with the findings of previous studies $[23,24$, 26]. These results indicate that young LARC patients have more severe pathological features than those of older patients.

Age is an important factor for survival benefits and the risk of complications in cancer patients following CRT. To clarify the influence of age on tumor response to NCRT in LARC patients, we performed a logistic regression analysis. pCR has been proposed as a surrogate endpoint for the efficacy of NCRT and oncological outcome in LARC patients. Our results demonstrated that young age is an independent predictive factor for $\mathrm{pCR}$ in LARC patients, as well as tumor size, pre-NCRT clinical N stage, and post-NCRT CEA level. Our results also indicated that young LARC patients have poor responses to NCRT, in terms of lower pCR rates. Therefore, more chemotherapy treatment options could be considered in the NCRT regimens in young LARC patients to increase the $\mathrm{pCR}$ rate. We further developed a nomogram for predicting $\mathrm{pCR}$ to facilitate the decision-making regarding organ-preserving strategies. Our results showed that decisions based on the $\mathrm{PCR}$ predictive nomogram yielded more favorable clinical consequences than the treat-all patient and treat-none schemes, even given an extremely small probability threshold. Additionally, the nomogram including age group had superior predictive capability than the nomogram excluding age group.

CSCs are the tumor-initiating cells that are responsible for tumorigenesis [30, 31]. Accumulating evidence has demonstrated that CSCs contribute to resistance to either chemotherapy or radiotherapy in various cancers, including rectal cancer [32-34]. It has been reported that cells expressing CD133, which is a putative marker of CSCs, are more resistant to radiochemotherapy than CD133- tumor cells in rectal cancer [32, 33]. Thus, CD133 expression in the tumor cells was detected as a CSC biomarker in LARC patients. However, the association between CSCs and treatment response in young LARC patients remains unclear. Herein, we hypothesized that young LARC patients have more CSCs, resulting in the resistance to NCRT. In this study, we demonstrated higher CD133 expression in cancer tissue of young LARC patients before NCRT compared with that in the old group. Additionally, higher CD133 expression was correlated with a worse prognosis. Together, these findings suggest that young LARC patients have a higher
CD133+ CSC burden, contributing to the lower pCR rates. Nevertheless, prospective studies with large sample sizes are required to confirm the role of CD133 in resistance to NCRT.

Several limitations of our study should be noted. First, our retrospective study was subject to potential selection bias. Second, age-related comorbidities, such as the Charlson comorbidity index, were not evaluated due to the lack of adequate data. Third, the impact of gene profiling on response to NCRT was not assessed owing to the lack of complete medical records in some cases. Fourth, CD133 expression was assessed only in some patients owing to the lack of adequate pretreatment specimens. Nevertheless, our study adds to our understanding of the impact of young age on the efficacy of NCRT in patients with LARC.

\section{Conclusions}

In this cohort study of 901 LARC patients treated at a single high-volume cancer center, young age ( $<40$ years) was identified as a significant determinant for predicting pCR in LARC patients following NCRT. Moreover, for the first time, we have demonstrated that young patients have a higher proportion of CSCs (CD133+) than older patients. Larger-scale prospective studies are warranted to confirm our findings.

\section{Abbreviations}

NCRT: Neoadjuvant chemoradiotherapy; LARC: Locally advanced rectal cancer; CRC: Colorectal cancer; TME: Total mesorectal excision; CEA: Carcinoembryonic antigen; MRI: Magnetic resonance imaging; pCR: Pathological complete response; ASA: American society of anesthesiology; CRM: Circumferential resection margin; HR: Hazard ratio; DFS: Disease free survival

\section{Acknowledgments}

The authors thank all the staff in Department of colorectal surgery, Fujian Medical University Union Hospital, Fuzhou, Fujian Province, People's Republic of China.

\section{Authors' contributions}

YYZ and GXG participated in all experimental work. XL and LLY collected the data. WY and MFX analyzed the data. All the authors drafted the paper and approved the final manuscript. All authors contributed toward data analysis, drafting and revising the paper and agree to be accountable for all aspects of the work.

\section{Funding}

This study was supported by the Science Foundation of the Fujian Province, (No. 2019 J01161), Special Financial Foundation of Fujian Provincial (No.20151297), the Startup Fund for Scientific Research, Fujian Medical University (2018QH2027, 2018S0130) and Professor Development Foundation of Fujian Medical University (No.JS11006).

\section{Availability of data and materials}

The data generated or analysed during this study are available from the corresponding author upon reasonable request.

\section{Ethics approval and consent to participate}

All had patients provided written informed consent for the scientific use of the clinical tissue samples informed consent for inclusion before they participated in the study. The study was conducted in accordance with the 
Declaration of Helsinki, and the protocol was approved by the Ethics Committee of Fujian Medical University Union Hospital.

\section{Consent for publication}

Not applicable.

\section{Competing interests}

The authors declare that they have no conflict of interest.

\section{Author details}

${ }^{1}$ Department of Colorectal Surgery, The First Affiliated Hospital of Fujian Medical University, Fuzhou, China. ${ }^{2}$ Department of Cardiac Surgery, Fujian Medical University Union Hospital, Fuzhou, China. ${ }^{3}$ Department of Pathology, Fujian Medical University Union Hospital, Fuzhou, China.

\section{Received: 30 December 2019 Accepted: 27 August 2020}

\section{Published online: 05 September 2020}

\section{References}

1. Siegel RL, Miller KD, Jemal A. Cancer statistics, 2017. CA Cancer J Clin. 2017; 67:7-30.

2. Cai X, Wu H, Peng J, Zhu J, Cai S, Cai G, Zhang Z. Tolerability and outcomes of radiotherapy or chemoradiotherapy for rectal cancer in elderly patients aged 70 years and older. Radiat Oncol. 2013;8:86.

3. Rutten $H$, den Dulk M, Lemmens V, Nieuwenhuijzen G, Krijnen P, JansenLandheer M, van de Poll FL, Coebergh JW, Martijn H, Marijnen C, van de Velde C. Survival of elderly rectal cancer patients not improved: analysis of population based data on the impact of TME surgery. Eur J Cancer. 2007:43:2295-300.

4. Patel SG, Ahnen DJ. Colorectal Cancer in the young. Curr Gastroenterol Rep. 2018:20:15.

5. O'Connell JB, Maggard MA, Liu JH, Etzioni DA, Ko CY. Are survival rates different for young and older patients with rectal cancer. Dis Colon Rectum. 2004;47:2064-9.

6. Gallagher EG, Zeigler MG. Rectal carcinoma in patients in the second and third decades of life. Am J Surg. 1972;124:655-9.

7. Roh MS, Colangelo LH, O'Connell MJ, Yothers G, Deutsch M, Allegra CJ, Kahlenberg MS, Baez-Diaz L, Ursiny CS, Petrelli NJ, Wolmark N. Preoperative multimodality therapy improves disease-free survival in patients with carcinoma of the rectum: NSABP R-03. J Clin Oncol. 2009;27:5124-30.

8. Sauer R, Liersch T, Merkel S, Fietkau R, Hohenberger W, Hess C, Becker H, Raab HR, Villanueva MT, Witzigmann H, Wittekind C, Beissbarth T, Rödel C. Preoperative versus postoperative chemoradiotherapy for locally advanced rectal cancer: results of the German CAO/ARO/AIO-94 randomized phase III trial after a median follow-up of 11 years. J Clin Oncol. 2012;30:1926-33.

9. van Gijn W, Marijnen CA, Nagtegaal ID, Kranenbarg EM, Putter H, Wiggers T, Rutten HJ, Påhlman L, Glimelius B, van de Velde CJ. Preoperative radiotherapy combined with total mesorectal excision for resectable rectal cancer: 12-year follow-up of the multicentre, randomised controlled TME trial. Lancet Oncol. 2011;12:575-82.

10. Park IJ, You YN, Agarwal A, Skibber JM, Rodriguez-Bigas MA, Eng C, Feig BW, Das P, Krishnan S, Crane CH, Hu CY, Chang GJ. Neoadjuvant treatment response as an early response indicator for patients with rectal cancer. J Clin Oncol. 2012;30:1770-6.

11. Maas M, Nelemans PJ, Valentini V, Das P, Rödel C, Kuo LJ, Calvo FA, GarcíaAguilar J, Glynne-Jones R, Haustermans K, Mohiuddin M, Pucciarelli S, Small W, Suárez J, Theodoropoulos G, Biondo S, Beets-Tan RG, Beets GL. Longterm outcome in patients with a pathological complete response after chemoradiation for rectal cancer: a pooled analysis of individual patient data. Lancet Oncol. 2010;11:835-44.

12. Neugut Al, Fleischauer AT, Sundararajan V, Mitra N, Heitjan DF, Jacobson JS, Grann VR. Use of adjuvant chemotherapy and radiation therapy for rectal cancer among the elderly: a population-based study. J Clin Oncol. 2002;20:2643-50.

13. Cafiero F, Gipponi M, Lionetto R. Randomised clinical trial of adjuvant postoperative RT vs. sequential postoperative RT plus 5-FU and levamisole in patients with stage II-III resectable rectal cancer: a final report. J Surg Oncol. 2003;83:140-6.

14. Vermeulen L. de Sousa e Melo F, Richel DJ, Medema JP. The developing cancer stem-cell model: clinical challenges and opportunities. Lancet Oncol. 2012;13:e83-9.

15. Boman BM, Wicha MS. Cancer stem cells: a step toward the cure. J Clin Oncol. 2008;26:2795-9.
16. Dick JE. Looking ahead in cancer stem cell research. Nat Biotechnol. 2009; 27:44-6.

17. Ryan R, Gibbons D, Hyland JM, Treanor D, White A, Mulcahy HE, O'Donoghue DP, Moriarty M, Fennelly D, Sheahan K. Pathological response following long-course neoadjuvant chemoradiotherapy for locally advanced rectal cancer. Histopathology. 2005;47:141-6.

18. Zhang Y, Xu Z, Sun Y, Chi P, Lu X. Knockdown of KLK11 reverses oxaliplatin resistance by inhibiting proliferation and activating apoptosis via suppressing the PI3K AKT signal pathway in colorectal cancer cell. Onco Targets Ther. 2018;11:809-21.

19. Rousson V, Zumbrunn T. Decision curve analysis revisited: overall net benefit, relationships to ROC curve analysis, and application to case-control studies. BMC Med Inform Decis Mak. 2011;11:45.

20. Vickers AJ, Elkin EB. Decision curve analysis: a novel method for evaluating prediction models. Med Decis Mak. 2006;26:565-74.

21. Camp RL, Dolled-Filhart M, Rimm DL. X-tile: a new bio-informatics tool for biomarker assessment and outcome-based cut-point optimization. Clin Cancer Res. 2004;10:7252-9.

22. Barbaro B, Leccisotti L, Vecchio FM, Di MM, Serra T, Salsano M, Poscia A, Coco C, Persiani R, Alfieri S, Gambacorta MA, Valentini V, Giordano A, Bonomo L. The potential predictive value of MRI and PET-CT in mucinous and nonmucinous rectal cancer to identify patients at high risk of metastatic disease. Br J Radiol. 2017;90:20150836.

23. Reggiani BL, Lionti S, Domati F, Pagliani G, Mattioli E, Barresi V. Histological grading based on poorly differentiated clusters is predictive of tumour response and clinical outcome in rectal carcinoma treated with neoadjuvant chemoradiotherapy. Histopathology. 2017;71:393-405.

24. Sengul N, Wexner SD, Woodhouse S, Arrigain S, Xu M, Larach JA, Ahn BK, Weiss EG, Nogueras JJ, Berho M. Effects of radiotherapy on different histopathological types of rectal carcinoma. Color Dis. 2006;8:283-8.

25. Grillo-Ruggieri F, Mantello G, Berardi R, Cardinali M, Fenu F, lovini G, Montisci M, Fabbietti L, Marmorale C, Guerrieri M, Saba V, Bearzi I, Mattioli R, Bonsignori M, Cascinu S. Mucinous rectal adenocarcinoma can be associated to tumor downstaging after preoperative chemoradiotherapy. Dis Colon Rectum. 2007;50:1594-603.

26. Shin US, Yu CS, Kim JH, Kim TW, Lim SB, Yoon SN, Yoon YS, Kim CW, Kim JC. Mucinous rectal cancer: effectiveness of preoperative chemoradiotherapy and prognosis. Ann Surg Oncol. 2011;18:2232-9.

27. Yu SK, Chand M, Tait DM, Brown G. Magnetic resonance imaging defined mucinous rectal carcinoma is an independent imaging biomarker for poor prognosis and poor response to preoperative chemoradiotherapy. Eur J Cancer. 2014;50:920-7

28. Oberholzer K, Menig M, Kreft A, Schneider A, Junginger T, Heintz A, Kreitner KF, Hötker AM, Hansen T, Düber C, Schmidberger H. Rectal cancer: mucinous carcinoma on magnetic resonance imaging indicates poor response to neoadjuvant chemoradiation. Int J Radiat Oncol Biol Phys. 2012;82:842-8.

29. Li Q, Cai G, Li D, Wang Y, Zhuo C, Cai S. Better long-term survival in young patients with non-metastatic colorectal cancer after surgery, an analysis of 69,835 patients in SEER database. PLoS One. 2014;9:e93756.

30. Papaccio F, Paino F, Regad T, Papaccio G, Desiderio V, Tirino V. Concise review: Cancer cells, Cancer stem cells, and Mesenchymal stem cells: influence in Cancer development. Stem Cells Transl Med. 2017;6:2115-25.

31. Ramos EK, Hoffmann AD, Gerson SL, Liu H. New opportunities and challenges to defeat Cancer stem cells. Trends Cancer. 2017;3:780-96.

32. Sprenger T, Conradi LC, Beissbarth T, Ermert H, Homayounfar K, Middel $P$, Rüschoff J, Wolff HA, Schüler P, Ghadimi BM, Rödel C, Becker H, Rödel F, Liersch T. Enrichment of CD133-expressing cells in rectal cancers treated with preoperative radiochemotherapy is an independent marker for metastasis and survival. Cancer. 2013;119:26-35.

33. Saigusa S, Tanaka K, Toiyama Y, Yokoe T, Okugawa Y, loue Y, Miki C, Kusunoki M. Correlation of CD133, OCT4, and SOX2 in rectal cancer and their association with distant recurrence after chemoradiotherapy. Ann Surg Oncol. 2009:16:3488-98.

34. Fan CW, Chen T, Shang YN, Gu YZ, Zhang SL, Lu R, OuYang SR, Zhou X, Li Y, Meng WT, Hu JK, Lu Y, Sun XF, Bu H, Zhou ZG, Mo XM. Cancer-initiating cells derived from human rectal adenocarcinoma tissues carry mesenchymal phenotypes and resist drug therapies. Cell Death Dis. 2013;4:e828.

\section{Publisher's Note}

Springer Nature remains neutral with regard to jurisdictional claims in published maps and institutional affiliations. 